\title{
Fabrication of Tailored Bragg Gratings by the Phase Mask Dithering/Moving Technique
}

\author{
Miguel MELO ${ }^{1}$ and Paulo V. S. MARQUES ${ }^{1,2^{*}}$ \\ ${ }^{1}$ Department of Physics and Astronomy, Faculty of Sciences, University of Porto, Rua do Campo Alegre 687, 4169-007 \\ Porto, Portugal \\ ${ }^{2}$ INESC TEC, Rua do Campo Alegre 687, 4169-007 Porto, Portugal \\ *Corresponding author: Paulo V. S. MARQUES $\quad$ E-mail: psmarque@fc.up.pt
}

\begin{abstract}
The most relevant aspects related to the phase mask dithering/moving method for the fabrication of complex Bragg grating designs are reviewed. Details for experimental implementation of this technique is presented, including theoretical analysis of the calibration functions for the correct dither/displacement. Results from tailored Bragg grating structures fabricated by this method are shown. Apodized Bragg gratings with modeled spatial profiles were implemented, resulting in side mode suppression levels of more than $20 \mathrm{~dB}$ in gratings showing transmission filtering level higher than $30 \mathrm{~dB}$. Chirped gratings with the spectral bandwidth up to $4 \mathrm{~nm}, \pi$-shift and sampled Bragg gratings with equalized peaks equally spaced by $0.8 \mathrm{~nm}(100 \mathrm{GHz})$ were also fabricated.
\end{abstract}

Keywords: Bragg gratings, optical fiber filters, optical fiber communications, optical fiber sensors

Citation: Miguel MELO and Paulo V. S. MARQUES, "Fabrication of Tailored Bragg Gratings by the Phase Mask Dithering/Moving Technique," Photonic Sensors, vol. 3, no. 1, pp. 81-96, 2013.

\section{Introduction}

Bragg gratings are key devices in the optical fiber sensors field. Several techniques for their fabrication have been demonstrated, starting with the internal writing [1] and the holographic method [2], for which two overlapping light beams create a periodic interference pattern. Other interferometric methods based on the standard Talbot arrangement were proposed [3, 4], enabling grating inscription with a wide wavelength tunability achieved simply by the rotation of two mirrors. Another fabrication method is the point-by-point $(\mathrm{PbP})$ technique and was first demonstrated in the early 1990s using an ultraviolet (UV) excimer laser [5, 6]. Each point (or each index perturbation) can be controlled in terms of the UV dose, dimensions and in terms of waveguide positioning when illuminated, and so custom designs can be achieved. However, the field received little interest at the time since it was a tediously long process and extremely difficult to focus a "clean" slit of UV light of sub-micron dimensions. It was almost one decade later, with the advent of femtosecond (fs) laser materials processing, for which the use of fs pulses enabled the inscription of highly localized microvoid modifications via nonlinear photoionization processes, that the $\mathrm{PbP}$ method started to show highly interesting potential. Directly, point-by-point inscription of fiber Bragg gratings by the infrared fs laser was reported for the first time by Martinez et al. [7]. A big advantage of this technique is that the fs

Received: 30 July 2012 / Revised version: 10 August 2012

(C) The Author(s) 2012. This article is published with open access at Springerlink.com 
laser-material interaction enables grating inscription into almost any fiber material without requiring photosensitivity. Also, due to the structural modification of the glass, these gratings present high-temperature stability. More recently, other complex grating designs were shown using the $\mathrm{PbP}$ method $[8,9]$.

Nevertheless, the mostly used and attractive technique is still the phase mask technique [10-13], since it greatly simplifies the fabrication process, while yielding high performance gratings. When compared with the holographic technique, the phase mask method offers easier alignment of the waveguide, reduced stability requirements on the photoimprinting apparatus and lower coherence requirements on the ultraviolet laser beam. Therefore, this technique allows Bragg gratings to be written with relaxed tolerances on the coherence of the writing beam and with better repeatability. The main drawback is that the grating period and profile are imposed by the fixed phase mask patterns, requiring different masks for different gratings. However, some fine wavelength tuning $(\sim 2 \mathrm{~nm})$ is possible when different mechanical tension levels are applied to the fiber during the photoinscription process [14].

Various types of Bragg gratings can be fabricated using the above mentioned methods. Desired spectral and dispersive characteristics can be achieved by varying numerous physical parameters like induced refractive index change, length, periodicity and fringe tilt. In this way, special structures like apodized, chirped, phase shifted and sampled gratings can be fabricated, instead of the standard uniform Bragg gratings. Most of the designed gratings for demanding practical applications are not uniform.

The use of a standard uniform phase mask for grating fabrication results in a uniform refractive index modulation along the grating length and in expected strong side lobes in the spectral response of Bragg gratings, undesirable in many applications like wavelength division multiplexing (WDM). The suppression of these secondary maxima can be achieved if the profile of the index modulation along the grating length is a given bell-like functional shape [15]. This procedure, called apodization, has been extensively studied and demonstrated [16-19]. Although apodization can be achieved simply by varying the intensity of the UV writing beam along the grating length, the accompanying variation in the average effective refractive index induces an undesired chirp that needs to be compensated by another exposure step through an amplitude mask [17]. Alternatively, complex phase masks with variable diffraction efficiency can be used to produce pure apodization $[18,20]$, or techniques based on periodic fiber stretching during exposure [21]. Another approach is the moving fiber/scanning beam, where pure apodization is achieved by the application of a variable dither to the fiber during the photoinscription process $[22,23]$. This technique has the major advantage of inducing a constant average refractive index change, since the average UV fluence is constant along the grating length. It also enables the creation of more complex structures, like chirped and phase shifted gratings, by executing a proper movement of the fiber while the beam scanning is being performed [23].

The information about fabrication methods of these complex structures is frequently dispersed in many key papers. This is particularly true for the case of fabrication employing phase mask dithering. Although this technique is very attractive, only a few of the intimate technological details have been published. This paper describes in a comprehensive manner the method and its potential through the demonstration of several types of tailored and complex Bragg gratings.

\section{Bragg gratings: the phase mask dithering/moving technique}

A Bragg grating is a periodic perturbation of the refractive index along the waveguide axis formed by 
exposure to an intense ultraviolet optical interference pattern. For example, in an optical fiber, the exposure induces a permanent refractive index change in the core of the fiber, by the mechanism of photosensitivity [24-26]. The effective refractive index for each grating position can be described by $n_{\text {eff }}(z)=n_{\text {eff }}^{0}+\delta n_{\text {eff }}(z)$, where $n_{\text {eff }}^{0}$ is the effective refractive index of the guided mode without UV induced perturbation, and $\delta n_{\text {eff }}(z)$ is given by [27]

$$
\delta n_{\mathrm{eff}}(z)=\overline{\Delta n}\left\{1+V(z) \cos \left[\frac{2 \pi}{\Lambda} z+\phi(z)\right]\right\}
$$

where $\overline{\Delta n}$ is the "dc" index change spatially averaged over a grating period, $V(z)$ is the "fringe visibility" of the index change, $\Lambda$ is the nominal grating period, and $\phi(z)$ describes grating chirp. The possibility to induce variations in the visibility [through $V(z)$ ] and/or chirp [through $\phi(z)$ ], combined or independently, can result in very complex structures, originating the desired spectral and temporal responses.

The phase mask method was reported as an improved technique for the fabrication of Bragg gratings [10, 11], allowing easier alignment and inscription when compared to interferometric methods. The UV light beam is spatially phase modulated and diffracted by the phase mask, as shown in Fig. 1. The produced interference pattern is then used to photoimprint a refractive index modulation in the photosensitive fiber placed in proximity and parallel behind the phase mask. As it can be seen from Fig. 1, the phase mask is basically a grating etched in a high quality silica plate with the well controlled space ratio and depth of the etched grooves. The principle of operation is based on the diffraction of an incident UV beam into several orders, $m=0, \pm 1, \pm 2, \cdots$.

The incident and diffracted orders satisfy the general diffraction equation, with the period of the phase mask, $\Lambda_{\mathrm{pm}}$, given by

$$
\Lambda_{\mathrm{pm}}=\frac{m \lambda_{\mathrm{UV}}}{\sin \left(\frac{\theta_{m}}{2}\right)-\sin \left(\theta_{i}\right)}
$$

where $\lambda_{\mathrm{UV}}$ is the wavelength of the writing radiation, $\theta_{m} / 2$ is the angle of the diffracted order, and $\theta_{i}$ is the angle of the incident UV beam. Each phase mask has a zero order minimized for a single incident wavelength. The interference pattern generated by the \pm 1 orders has a period $\Lambda_{g}$, which is given by

$$
\Lambda_{g}=\frac{\lambda_{\mathrm{UV}}}{2 \sin \left(\frac{\theta_{m}}{2}\right)}=\frac{\lambda_{\mathrm{UV}}}{2 \frac{\lambda_{\mathrm{UV}}}{\lambda_{\mathrm{pm}}}}=\frac{\Lambda_{\mathrm{pm}}}{2}
$$

which is exactly half the value of the phase mask period and is independent of the UV wavelength.

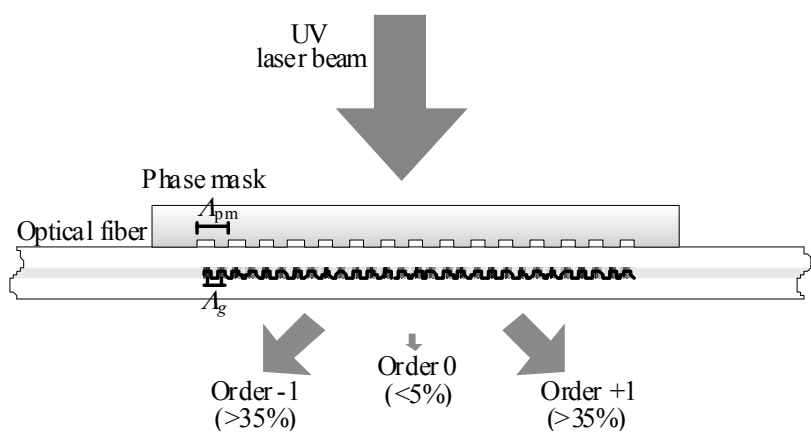

Fig. 1 Schematic diagram of the phase mask method.

The Bragg wavelength, $\lambda_{B}$, required for the grating in the waveguide is thus determined by the period of the grating etched in the mask, $\Lambda_{\mathrm{pm}}$, obeying to the well known Bragg's law condition:

$$
\lambda_{B}=n_{\text {eff }} \Lambda_{\mathrm{pm}} .
$$

A flexible writing method for fabrication of advanced gratings using a single exposure step can be implemented by dithering/moving the phase mask while the UV beam is scanning all the grating length. Basically, this method uses controlled dithering of the phase mask or the fiber/waveguide in order to control the local grating visibility and phase, without affecting the average refractive index. High dithering amplitudes destroy the interference pattern created by the phase mask at the fiber plane by fringe blurring. This corresponds to low visibility and results in gratings with low induced refractive index modulation amplitudes, i.e., gratings exhibiting low reflectivity. Dithering is thus an ideal mechanism for varying the local strength and/or phase of the grating without changing its local 
Bragg wavelength. Based on this, a technique called moving fiber/phase mask scanning method, which also allows tuning the Bragg wavelength, has been demonstrated [22]. The setup is similar to previous mask scanning configurations [12, 28], but with the difference that the fiber, or alternatively the phase mask, is slowly moved (but without dithering) while the incident UV beam is being scanned, overcoming many of the limitations associated with phase masks. For uniform motion of the mask and/or the fiber, the relative movement results in a shift in the Bragg wavelength. If $\lambda_{0}$ is the unshifted Bragg wavelength and $v_{f}$ and $v_{s c}$ are the fiber and scanning beam velocities (with $v_{f}<<v_{s c}$ ), respectively, the wavelength shift is given by [22]

$$
\Delta \lambda=\lambda_{0} \frac{v_{f}}{v_{s c}} .
$$

Thus, for a shift of about $1 \mathrm{~nm}$, the waveguide or phase mask must move at only $0.1 \%$ of the UV beam scanning velocity. An important aspect that should be taken into account is that for larger wavelength shifts, the grating strength decreases, since the refractive index modulation is averaged when the fiber moves too quickly across the interference pattern formed by the mask. The grating reflectivity, $R$, decreases with the wavelength shift as [22]

$$
R \sim \operatorname{sinc}^{2}\left(\frac{\pi D v_{f}}{\Lambda_{g} v_{s c}}\right)=\operatorname{sinc}^{2}\left(\frac{2 n_{\mathrm{eff}} \pi D \Delta \lambda}{\lambda_{0}^{2}}\right)
$$

where $D$ is the writing beam diameter, and $n_{\text {eff }}$ is the effective refractive index $\left(\lambda_{0}=2 n_{\text {eff }} \Lambda_{g}\right)$. From (6), it can be seen that $R=0$ for $v_{f, \text { max }}= \pm \Lambda_{\mathrm{g}} v_{s c} / D$ or $\Delta \lambda_{\max }=\lambda_{0} / 2 n_{\text {eff }} D$. Therefore, if the initial waveguide or phase mask velocity is set to $+v_{f, \text { max }}$ and linearly decreased to $-v_{f, \max }$ during the course of writing the grating, the result will be a linearly chirped grating (across $2 \Delta \lambda_{\max }$ ), automatically apodized with a sinc profile.

The next sections will describe in detail the phase mask dithering/moving setup that was implemented for the fabrication of tailored gratings, including pure apodized, chirp and phase shifted gratings, either in optical fibers or in planar waveguides.

\section{Experimental arrangement}

\subsection{Setup description}

The essence of the method described here is based on the fact that the phase mask is moved/dithered during the UV beam scanning by the action of a piezoelectric transducer (PZT), overcoming the limitations associated with the use of uniform phase masks. The experimental setup for the gratings fabrication is shown in Fig. 2.

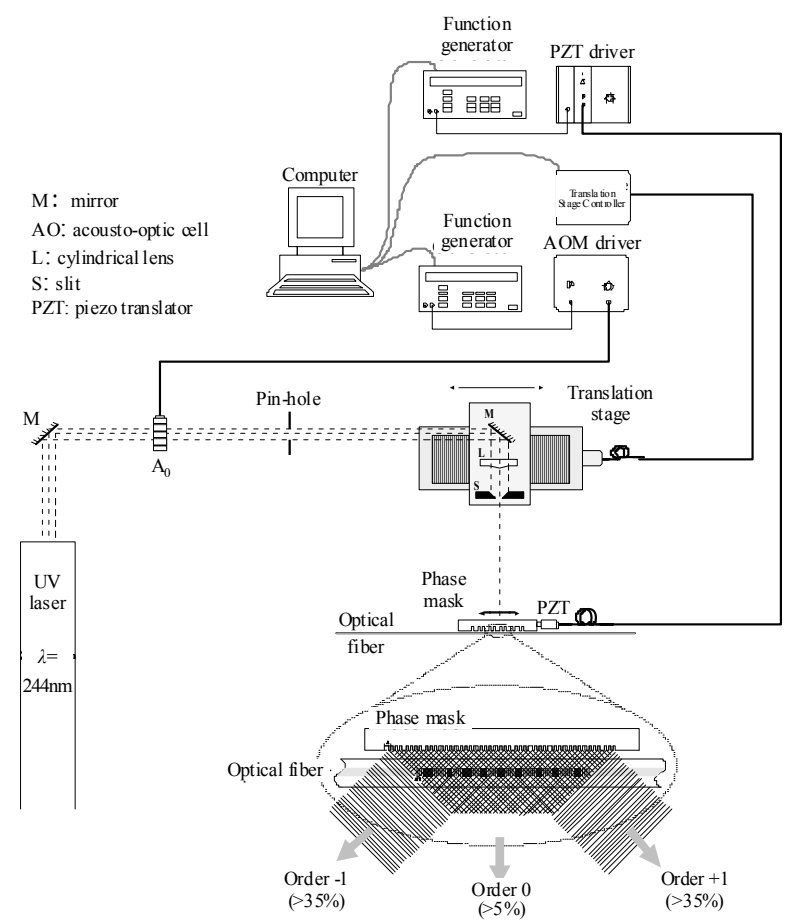

Fig. 2 Experimental setup for fiber/planar waveguide gratings photoinscription.

The optical source used for the photoinscription was a frequency doubled continous wave $(\mathrm{CW})$ Argon laser (Coherent Innova ${ }^{\circledR}$ Sabre $^{\circledR}$ Fred $^{\text {TM }}$ ) operating at $244 \mathrm{~nm}$ with a maximum output optical power of $500 \mathrm{~mW}$ at this wavelength. The UV laser beam ( $\sim 1-\mathrm{mm}$ diameter), was then directed to an acousto-optic modulator (AOM) by a mirror. The modulator (AA.MQ.80/A2 from A.A.-Automates et Automatismes) controlled the power passing through it by deflecting the incident beam from the 
order 0 to the order +1 according to the voltage applied to the cell by the AOM driver (AA.MOD.80-4W from A.A.-Automates et Automatismes). The maximum efficiency of the AOM was $85 \%$. The beam was then spatially "cleaned" by a pin-hole that also filtered the zero order. A mirror, a cylindrical lens and a slit were mounted on a translation stage in order to have the ability to scan the beam over different and desired grating lengths. A cylindrical lens $(f=30 \mathrm{~mm})$ was used to focus the beam on the photosensitive waveguide, and the slit, with the micrometer resolution, controlled the lateral dimension of the beam incident on the waveguide. The translation stage (Schneeberger TMF3 with power chassis TPP1/2) had a maximum resolution of $0.08 \mu \mathrm{m}$ and a maximum displacement distance of $20 \mathrm{~cm}$. The phase mask was placed on a proper machined aluminium holder which could be dithered and/or displaced by a coupled PZT. It was basically a two-arm deflector with enough stiffness to allow the mask to follow the PZT piston movement when it was dithered at frequencies in the order of the tens of Hz. The PZT (P-841.10 from PI-Physik Instrument), had a resolution of $0.3 \mathrm{~nm}$ and a maximum displacement length of $15 \mu \mathrm{m}$, and was controlled by an amplification low voltage PZT (LV-PZT) module (E-505.00) connected to a sensor module (E-509.X1). Both the PZT and the AOM drivers were controlled by independent synthesized function generators (Model DS345-30MHz from Stanford Research Systems).

The fiber or planar waveguide was then placed after the phase mask, in the focal plane of the lens. The sample was positioned with the help of a microscope, allowing the parallelism and distance between the sample and the phase mask to be adjusted at any time. In the case of optical fibers, the vertical alignment was performed simply by the visualization of the diffraction pattern on a target behind the fiber and when illuminated by the UV laser beam. A Michelson interferometer allowed the visualization (by the interference fringes displacement) of the dither/displacement of the phase mask, since one of the mirrors was placed in the two-arm deflector holder where the phase mask was fixed. The purpose was only to give a visual inspection of the phase mask behavior at each position along the grating, since any dither amplitude change was clearly identified. All the writing was computer controlled by a LabView ${ }^{\mathrm{TM}}$ based program developed for this purpose.

\subsection{Basic calibration}

It has been stated in the previous sections that the control of the grating modulation profile during writing was achieved by a precise control of the phase mask dithering amplitude for each position of the UV beam over the fiber. Correct adjustment of the dithering amplitude is then crucial to have a good control of the fringe visibility. This is easily achieved by changing the phase mask dither voltage as the writing beam scans across the fiber, and therefore one is able to control the index modulation written into the fiber at each position. So, the way how the dither voltage applied to the PZT affects the refractive index modulation amplitude is one of the most important aspects when implementing this technique in a particular writing system. To calculate the resultant refractive index change, one can start by considering the superposition of spatially dependent waves, with different phases, corresponding to the pattern created by the mask at the fiber plane during one dither period. Thus, any superposition of harmonic and coherent waves is given by

$$
\psi(x)=\sum_{i=1}^{N} A \cos \left(2 \pi \frac{(x+i \Delta x)}{\Lambda}\right)
$$

where $A$ corresponds to the amplitude of each wave, $\Lambda$ represents the period of each wave (and will correspond to the period of the resultant wave), and $x+i \Delta x$ is the position of wave $i$ (in the limit, $x+N \Delta x$ corresponds to the position of the wave resulting from the maximum displacement induced in the phase mask), which is by itself an harmonic wave. 
The amplitude variation of this resultant wave gives the information of the refractive index modulation that can be achieved. However, the calibration curve for how the grating amplitude varies with the dither amplitude depends also on the periodic function describing the oscillatory motion of the phase mask. As an example, the simulation of the square and the triangular dither functions will be presented using (7). For simplicity, the amplitude variation of the resultant wave is normalized and represented in terms of fringe visibility. The dither amplitude is represented in units of the grating period, $\Lambda_{g}$. For the square function, $N=2$ (representing two states of the square wave function in a period) and $\Delta x=$ dither amplitude, and normalized to a grating period, so that a dither amplitude of 0.5 corresponds to a displacement equal to half of a grating period. For the triangular function, $N=100$ and $\Delta x=$ dither amplitude $/ N$, which results in dividing a function period into 100 small sections, i.e., superposition of 100 harmonic waves, in order to simulate the continuous linear movement that this function induces, unlike the first case that is represented by a discrete two-step function. Also in this second case, a dither amplitude of 0.5 corresponds to a displacement equal to half of a grating period. Thus, Fig. 3 shows the fringe visibility versus the dither amplitude for both the square and triangular dither functions.

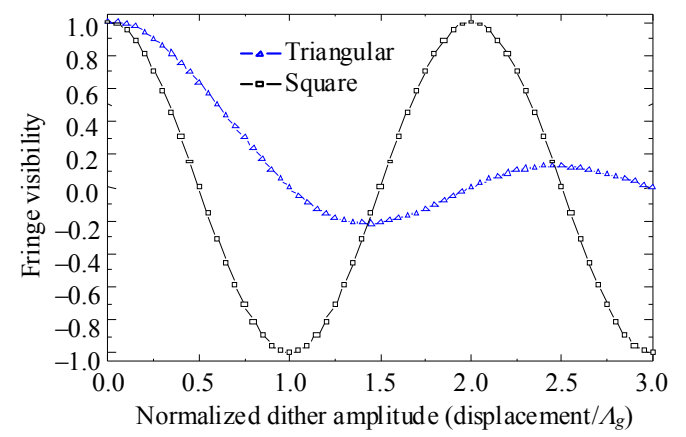

Fig. 3 Variation of fringe visibility with the dither amplitude (in units of the grating period) for the square and the triangular carrier functions.

As it can be confirmed from Fig. 3, as the amplitude of the dithering increases, the visibility of the grating fringes decreases until a certain value where the grating fringes disappear entirely; this point is referred to as the extinction amplitude. After this extinction point, the visibility of the fringes grows again, but now the written grating is 180 degrees out of phase relatively to its original position. This is known as over-dithering, and it produces a grating with an opposite phase to the one with the dither amplitude below the extinction point; this is shown by a negative fringe visibility. For the square wave dithering function, the visibility varies cosinusoidally with increasing the dither amplitude, while for the triangular dither function the visibility shows a sinc like behavior.

For this work, and in terms of the experimental application, the interest of the calibration curves shown in Fig. 3 was restricted to the positive fringe visibility values between dither amplitude points ranging from zero to one grating period. Also, for experimental applicability in this work, as shown latter on, the axes should be interchanged, and the dither amplitude displayed as a function of the fringe visibility, or as it will be called from now on, as a function of the normalized index modulation. This representation is shown in Fig. 4.

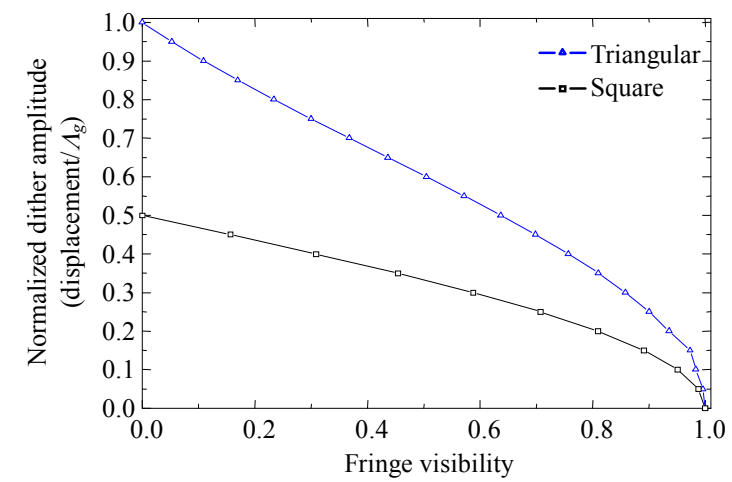

Fig. 4 Dither amplitude (in units of the grating period) as a function of the normalized modulation index for the square and the triangular carrier functions.

From Fig. 4, it can be seen that using a triangular carrier function, the extinction point, i.e., the dither amplitude value for which the modulation amplitude of the grating is completely destroyed, corresponds to one grating period; for square excitation, this 
value is half the grating period. For experimental applicability of these functions, a calibrated PZT was used, as seen in Fig. 2. Its calibration curve relates the displacement to the applied voltage.
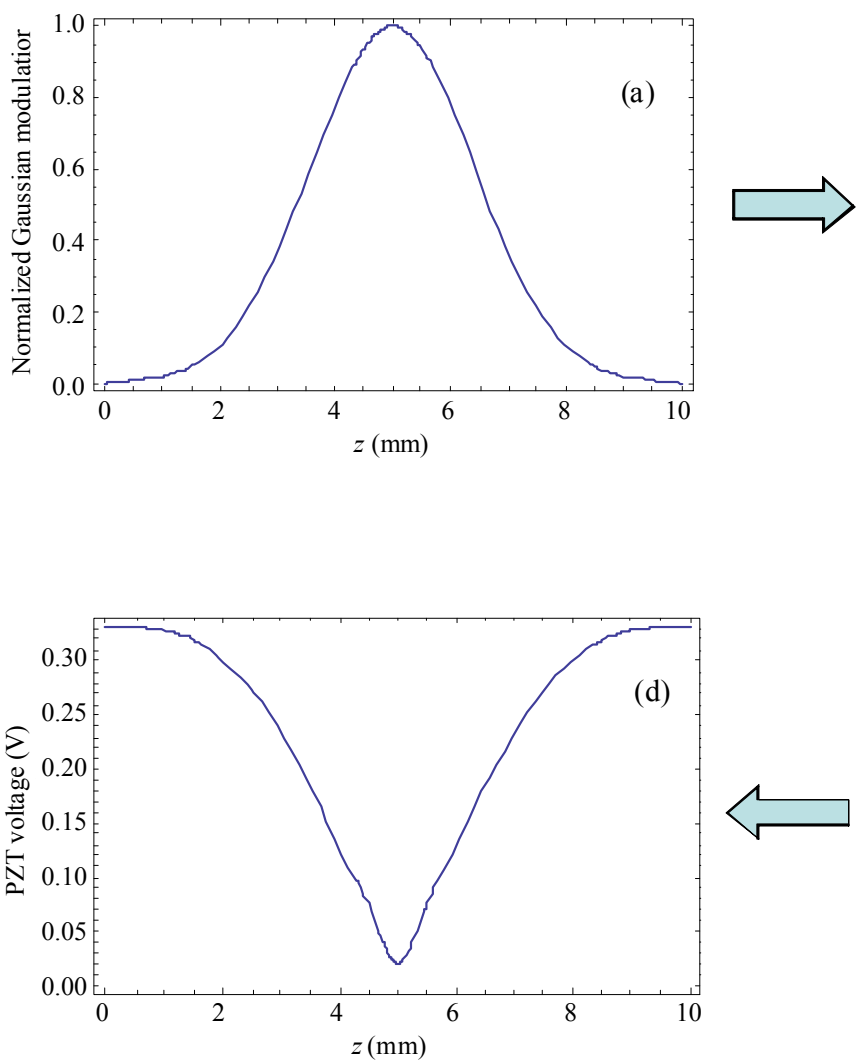

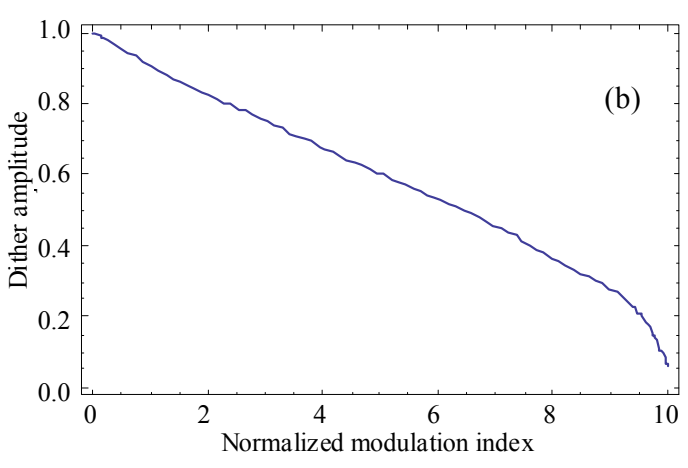

To finalize, and as an example, Fig. 5 shows how these calibration curves are integrated in the system. A Gaussian apodization profile and a triangular wave dithering function were used.

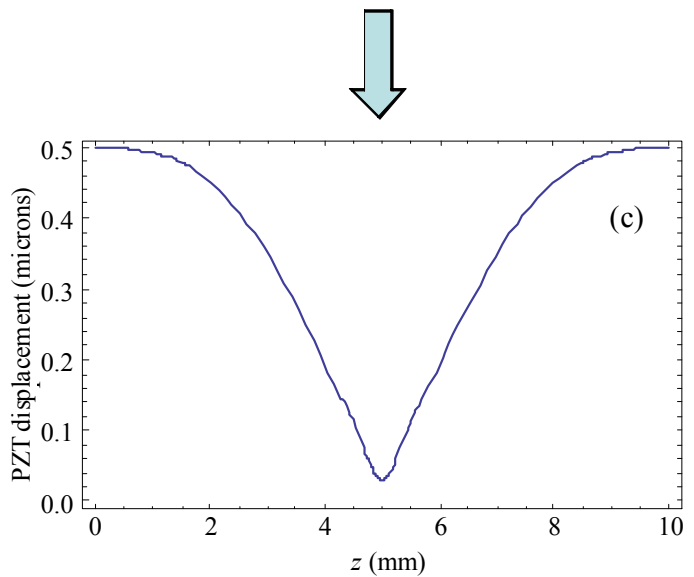

Fig. 5 Example of implementation of the Gaussian apodization profile using the required calibrations.

First of all, the desired spatial profile function should be introduced. In the case of Fig. 5, a Gaussian profile for a $10-\mathrm{mm}$ grating is displayed [Fig. 5(a)]. After having the normalized modulation profile [Fig. 5(b)], the PZT displacement (dithering amplitude) for each grating position can be derived by using one of the calibration functions showed in Fig. 4 (in this example, the triangular dithering function is used) - Fig. 5(c). Finally, using the PZT calibration curve (displacement vs voltage), the necessary voltage at each grating position is obtained - Fig. 5(d). As it can be seen, a peak-to-peak dithering amplitude of $0.34 \mathrm{~V}$ is needed to destroy the interference pattern (using this particular PZT) and wash out the modulation index at both grating ends. It should be stressed that there is not an objective to include effects related to materials non-linearity in what concerns the photosensitivity response under UV exposure. If non-linearity exists, its effect should be also included.

\section{Fabrication of Bragg gratings: experimental results}

\subsection{Phase mask dithering amplitude variation}

Using the calibration curve of Fig. 4 (with the triangular PZT driving function), several uniform Bragg gratings were written, changing the writing visibility between 0 and 1 . A visibility of 1 means that one should be able to produce a Bragg grating 
with the maximum reflectivity (corresponding to no dithering of the phase mask) and that for a visibility of 0 one should produce a grating with zero (or nearly zero) reflectivity. Figure 6 shows the obtained grating reflectivities for different visibilities.


Fig. 6 Several $30 \mathrm{~mm}$ long uniform fiber Bragg gratings fabricated with different phase mask dithering amplitudes.

All 30-mm-length uniform Bragg gratings were written in the hydrogen loaded standard single mode fiber (ITU-T G.652), with UV power of $50 \mathrm{~mW}$ incident in the phase mask (with a period of $1058.5 \mathrm{~nm}$ ) and a beam scanning velocity of $30 \mu \mathrm{m} / \mathrm{s}$. For each grating, the visibility changed from 0 to 1 , in steps of 0.25 . For the writing conditions employed, the strongest grating presented a peak reflectivity of about $96 \%$ and the weakest peak reflectivity of $0.3 \%$, showing that the system performed well.

\subsection{Apodized fiber Bragg gratings}

Implementation of analytical apodization functions is the most straightforward approach to achieve side lobes suppression in Bragg gratings. The Gaussian, the raised cosine and the sinc functions are among the most popular profiles [27,
29]. An experimental comparison between a uniform Bragg grating and the mentioned apodized Bragg gratings is shown in Fig. 7.

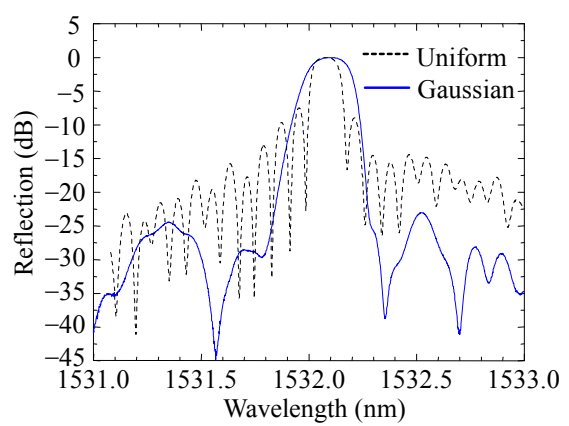

(a)

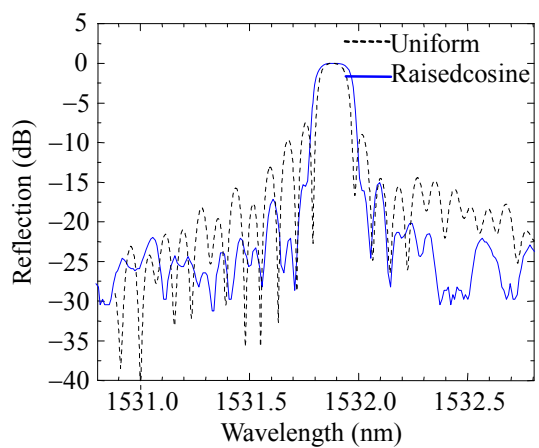

(b)

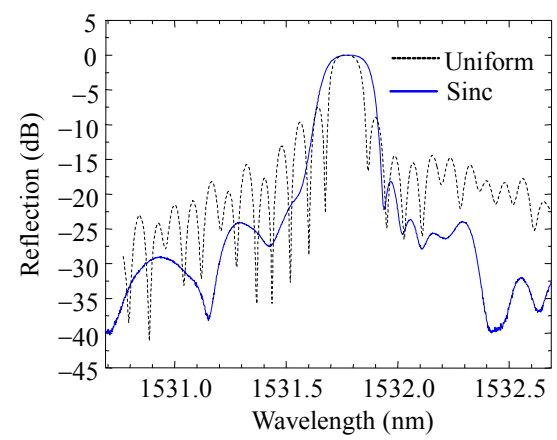

(c)

Fig. 7 Experimental comparison of a uniform Bragg grating and a (a) Gaussian, a (b) raised cosine, and a (c) sinc apodized Bragg grating.

All gratings were fabricated in a hydrogen loaded standard single mode fiber (ITU-T G.652), had peak reflectivities of around $90 \%$, and it is clearly seen that side lobe suppression was achieved for every case, when directly compared with the uniform grating. As it can be seen, the Gaussian apodization profile is the one that gives more side lobe suppression, while the raised cosine still 
presents a more clear side lobe structure, closer to the one obtained for the uniform case. Optimized performance of each grating can be achieved by optimizing the relevant parameters of the implemented functions, for example its full width at half maximum.

Sometimes and in order to achieve desired spectral and temporal responses of Bragg gratings, grating spatial profiles were generated by sophisticated synthesis techniques. Some examples are the genetic algorithm $[30,31]$ and the layer-peeling method [32-34]. The idea is to design the grating structure by numerical optimization using a certain merit function and a goal spectrum. Some modelled profiles using these techniques are shown in Fig. 8.

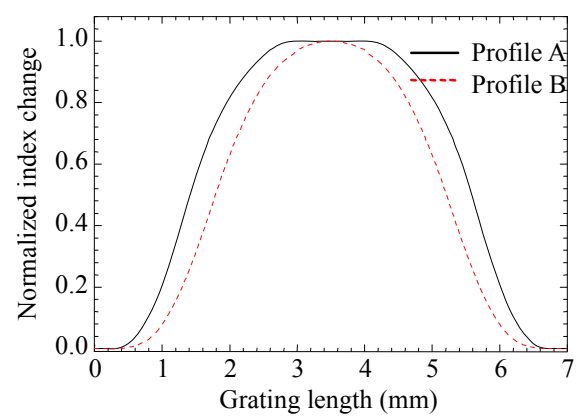

Fig. 8 Apodization spatial profiles provided by TUHH.

These profiles, supplied in a text file with two columns, the first indicating the grating position and the second indicating the normalized index change, were the input for the fabrication software. Using the profile $\mathrm{A}$, an $80 \%$ reflectivity grating was fabricated (Fig. 9).

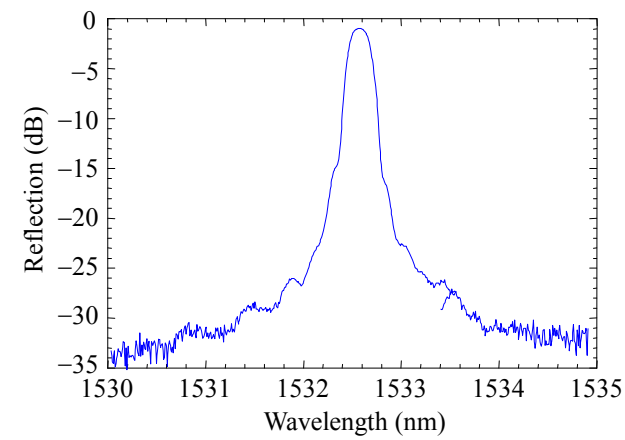

Fig. 9 Fiber Bragg grating fabricated by the spatial profile A shown in Fig. 8.
The grating of Fig. 9 had a measured transmission filtering level of about $6.7 \mathrm{~dB}(78 \%$ reflectivity), and the reflection spectra show that good side lobe suppression was achieved. To confirm that the results were according to the desired profile, reconstruction of the spatial distribution of the coupling coefficient was performed by TUHH - Technische Universität Hamburg - Harburg, using coherent optical frequency domain reflectometry (OFDR) [35], and the result is shown in Fig. 10.

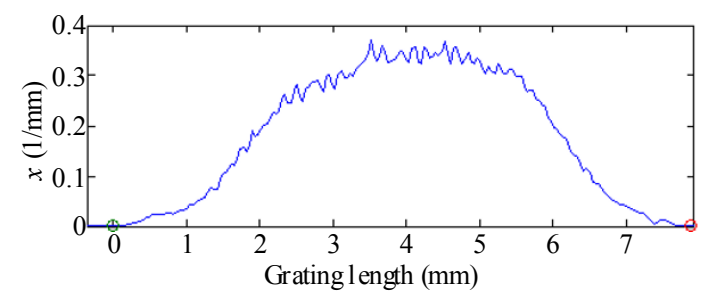

Fig. 10 Measured magnitude of $k_{a c}(z)$ of the grating of Fig. 9 using OFDR.

The peak reflectivity of the grating was $78 \%$ which corresponded to a calculated coupling coefficient of $0.39 \mathrm{~mm}^{-1}$. The magnitude of the coupling coefficient of the reconstructed grating is thus in good agreement with the calculated value, as well as the overall obtained shape profile when compared to the one of Fig. 8 (profile A).

To simultaneously achieve high reflectivity $(>99.9 \% \Rightarrow 30 \mathrm{~dB})$ and high side lobe suppression $(>25 \mathrm{~dB} @ \pm 0.6 \mathrm{~nm}) \quad$ suitable to the envisaged DWDM applications, the profile B on Fig. 8 was found. It was implemented in the experimental setup using hydrogen loaded fibers with high Germanium doping levels. The typical results obtained with the profile B represented in Fig. 8, are given in Fig. 11.

Both fiber Bragg gratings shown in Fig. 11 had a total length of $7 \mathrm{~mm}$ and were written with a scanning beam velocity of $30 \mu \mathrm{m} / \mathrm{s}$, but with different incident optical powers. Therefore, the grating (a) had a measured transmission filtering level of $25 \mathrm{~dB}$, while the grating (b) had a value of $30 \mathrm{~dB}$. The reflection spectra showed that the first side lobes appeared at levels above $20 \mathrm{~dB}$ and a 
noise floor situated at around $30 \mathrm{~dB}$ for both gratings. The first grating presented a flat-hat top of $0.3 \mathrm{~nm}$, while the second presented $0.4 \mathrm{~nm}$, both inside a $0.1-\mathrm{dB}$ power variation from the peak maximum.

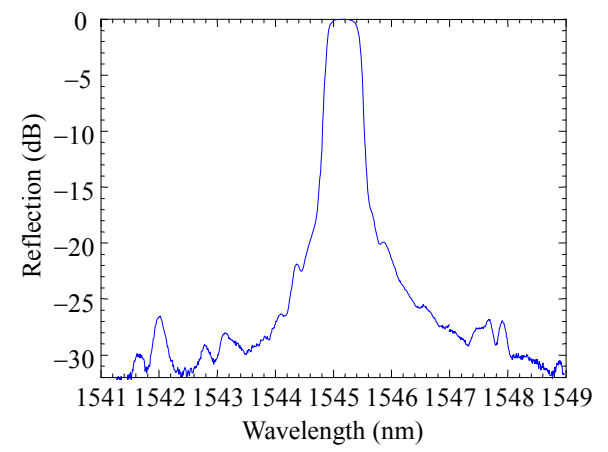

(a)

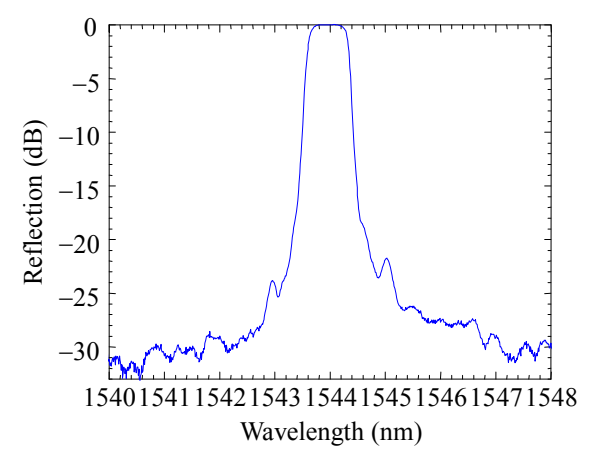

(b)

Fig. 11 High reflectivity apodized fiber Bragg gratings with (a) $25 \mathrm{~dB}$ and (b) $30 \mathrm{~dB}$ transmission filtering levels, using the spatial profile B of Fig. 8.

\subsection{Chirped fiber Bragg gratings}

In the case of chirped fiber Bragg gratings, the phase mask was slowly moved (unidirectionally) while the writing beam scanned the waveguide, allowing the production of variable wavelength/phase shifted gratings. This process enabled a gradual phase shift to be added to the grating while being written. For uniform motion, this resulted in a simple shift in the Bragg wavelength, as described by (5). To demonstrate this technique experimentally, five different Bragg gratings were written in the hydrogen loaded standard single mode fiber (ITU-T G.652) with a total length of $10 \mathrm{~mm}$ each and using a beam diameter of $350 \mu \mathrm{m}$. The scanning beam velocity was $10 \mu \mathrm{m} / \mathrm{s}$ for all gratings, while the phase mask velocity was different for each grating (no acceleration). Figure 12 shows the obtained results.

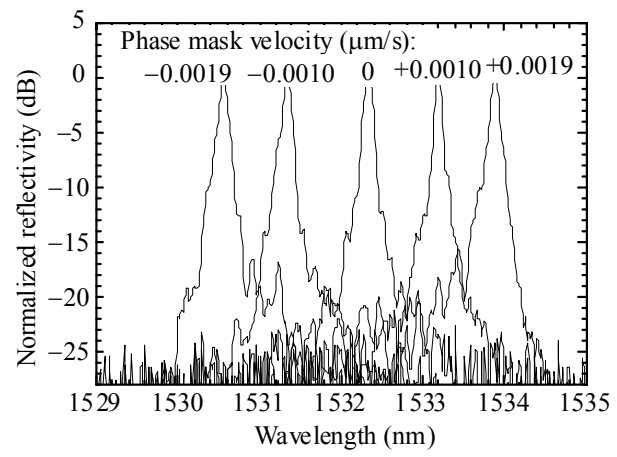

Fig. 12 Wavelength shift by the moving phase mask/scanning beam technique.

Shifts to the right of the normal Bragg wavelength (static phase mask) represent movement of the phase mask and beam scanning in the same direction, whereas shifts to shorter wavelengths represent movement of the phase mask and beam scanning in opposite direction. For each side, a wavelength shift of around $2 \mathrm{~nm}$ was achieved, limited by the PZT maximum displacement, resulting in a total wavelength tunability of $4 \mathrm{~nm}$. Another important aspect that it is not shown in Fig. 12 , since the graph is normalized, is that for larger wavelength shifts, the grating strength decreases, since the refractive index modulation decreases when the phase mask velocity increases. The grating reflectivity has the dependence already described by (6).

It is expectable that increasing the phase mask velocity linearly (constant acceleration) during the UV writing results in a chirped grating. The results are shown in Fig. 13. From here, one can confirm that, at least spectrally, the gratings produced are chirped. Also, Figs. 13(a) and 13(b) show the grating spectral evolution during the photoinscription. In the first case, the phase mask and the scanning beam move in opposite directions (negative chirp), while in the second their movements are in the same direction (positive chirp). Figure 13(c) is a combination of a negative chirp and a positive chirp, obtained by a double exposure process, maintaining 
all the writing parameters from one exposure to the other, except that the phase mask direction is reversed.

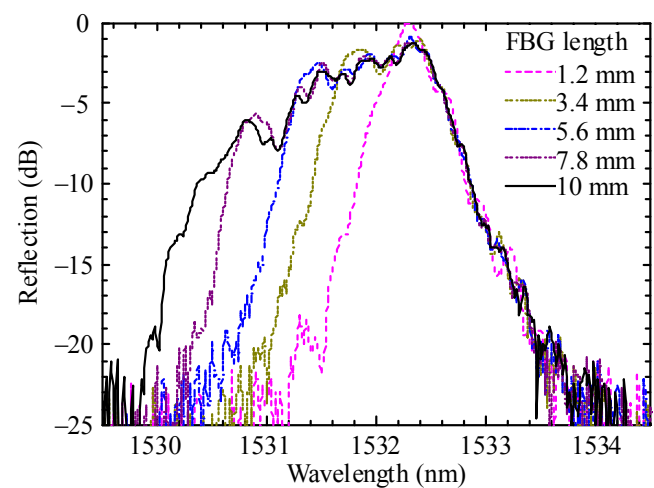

(a)



(b)

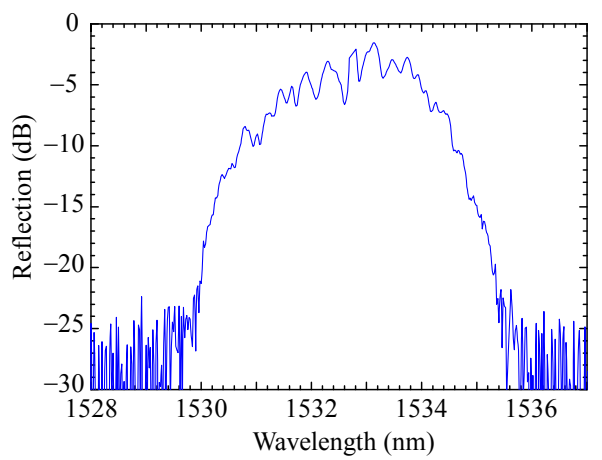

(c)

Fig. 13 Spectra of chirped fiber Bragg gratings fabricated by simultaneous phase mask movement and beam scanning: the spectra are obtained when the phase mask and beam move (a) in opposite directions (negative chirp), (b) in the same direction (positive chirp), and (c) in both (double exposure).

Obviously, it is expectable that the second exposure, if not compensated by higher fluence, results in a lower reflectivity since the photosensitivity of the fiber is already reduced by the first illumination process. Also clear from the figure is that the grating strength decreases for larger wavelength shifts, as expected. Using the modulated phase-shift method [36], the dispersion value measured for the chirped fiber Bragg grating of Fig. 13(a) was found to be $38.2 \mathrm{ps} / \mathrm{nm}$.

\subsection{Phase shifted fiber Bragg gratings}

A phase shifted grating consisted of a grating with an introduced phase jump in the refractive index modulation. This phase shift originated two Bragg gratings out of phase that acted like a resonant cavity. The relations between the path difference of the two Bragg gratings, $\delta l$, and the corresponding change in the phase, $\phi$, of an incident wave are well known and result in [37]

$$
\delta l=\frac{\lambda_{B}}{4 \pi n_{\mathrm{eff}}} \phi
$$

for normal incidence, which is the case.

The inscription of precise complex gratings, such as phase shifted structures, usually involves the translation of an interferogram by the desired phase shift. In our case, the shift of the interference pattern was achieved by translating the phase mask, being the precision with which the phase shift was made determined by the precision of the induced spatial shift along the waveguide, i.e., translation across the interferogram. Figure 14 shows a $\pi$-shift fiber Bragg grating fabricated by displacing the phase mask at the middle of the grating and its reflection spectra simulated by the transfer matrix method.

The 10-mm-length grating was fabricated in the hydrogen loaded standard single mode fiber (ITU-T G.652), using a phase mask with a period of $1058.5 \mathrm{~nm}$, and characterized with a resolution of $1 \mathrm{pm}$, using a tunable laser source to probe the grating and reading the reflected power on a power meter.

The experimental spectrum was fitted with a calculated profile from numerical simulation, with the following parameters: grating length of $10 \mathrm{~mm}$, refractive index change of $1.7 \times 10^{-4}$ and a spatial 
shift of $264.625 \mathrm{~nm}$, corresponding to $1 / 4$ of the phase mask pitch [as given by (8) and using (4)].

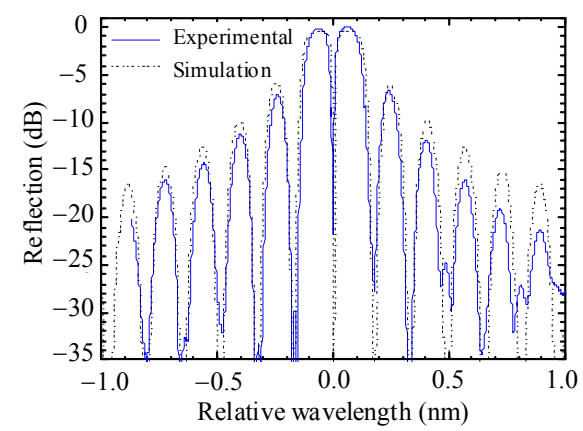

Fig. 14 Experimental result and simulation of a $\pi$-shift fiber Bragg grating.

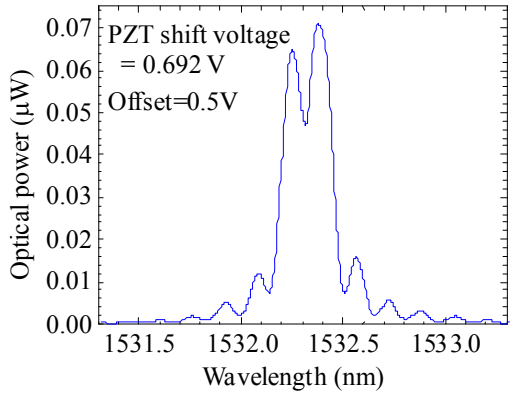

(a)

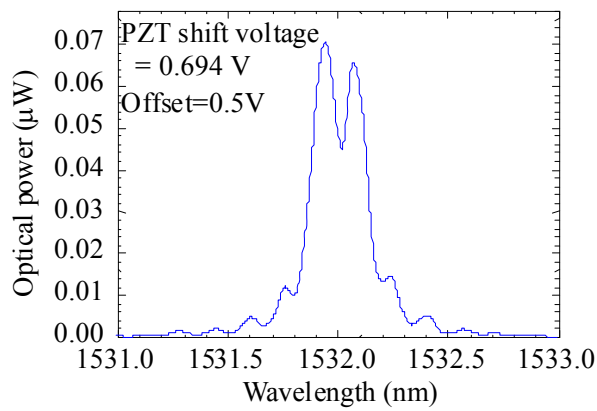

(b)

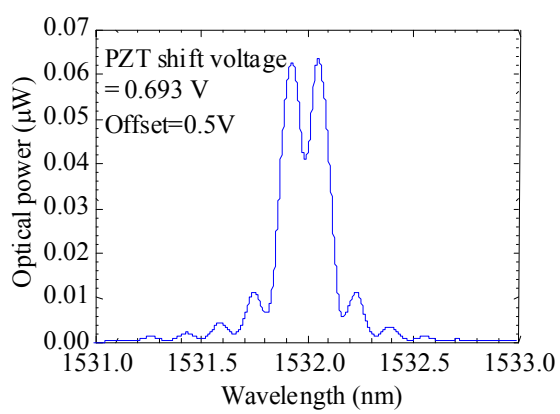

(c)

Fig. 15 Fine control of $\pi$-shift fiber Bragg gratings by changing the PZT driven voltage by $1 \mathrm{mV}$, corresponding to a spatial shift difference of $15 \mathrm{~nm}$.
In order to achieve fine control and also check calibrations, the PZT shift driven voltage was changed by its maximum allowable resolution $(1 \mathrm{mV})$. Three phase-shifted fiber Bragg gratings were then written with a difference of $1 \mathrm{mV}$ in the applied voltage, corresponding to a spatial shift difference of $15 \mathrm{~nm}$

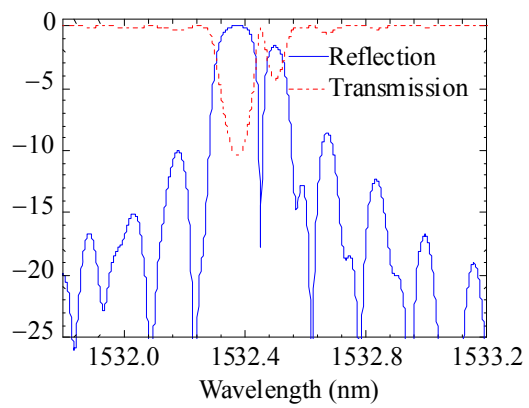

(a)

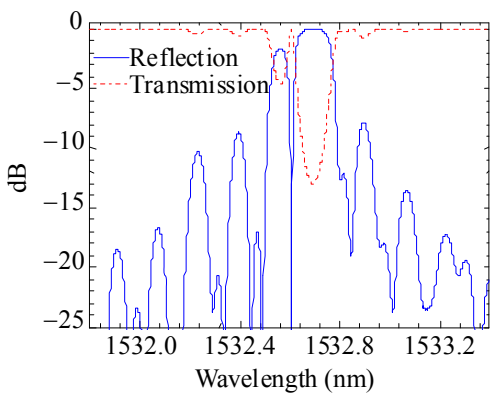

(b)

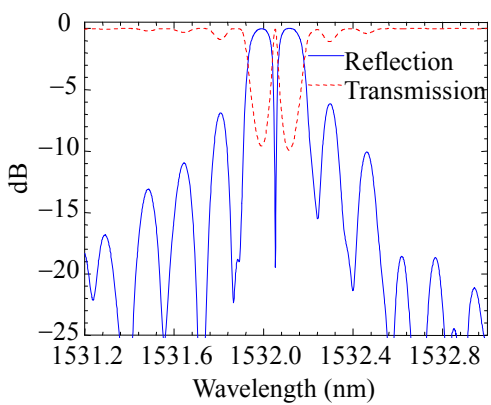

(c)

Fig. 16 Transmission and reflection spectra of 10-mm-length fabricated fiber Bragg gratings with a (a) $\pi / 2$, a (b) $3 \pi / 2$, and a (c) $\pi$ phase shift.

All fiber Bragg gratings shown with a total length of $10 \mathrm{~mm}$, were written with a scanning beam velocity of $30 \mu \mathrm{m} / \mathrm{s}$ and were fabricated in the hydrogen loaded standard single mode fiber (ITU-T G.652). The central resonant wavelength was not totally resolved since the characterization was 
carried out using a broadband source and an optical spectrum analyzer (OSA) with the maximum resolution of $0.05 \mathrm{~nm}$. Figure 15 shows that a good control of the position of resonance (or phase shift value) can be achieved allowing fine tuning. To test reproducibility and accuracy of the writing setup, three fiber Bragg gratings with the spatial shifts of $\Lambda_{\mathrm{pm}} / 8,3 \Lambda_{\mathrm{pm}} / 8$ and $\Lambda_{\mathrm{pm}} / 4$, corresponding to expected phase shifts of $\pi / 2,3 \pi / 2$ and $\pi$ [see (8) ] respectively, were written. Figure 16 shows the results obtained.

\subsection{Sampled fiber Bragg gratings}

More complex structures can be implemented by a periodic modulation of the refractive index amplitude and/or phase in the waveguide (sampled Bragg gratings). The resultant reflection spectrum and channel separation is a function of the shape and period of this modulation. The sampled grating is a conventional grating at the appropriate wavelength multiplied by a sampling function. Therefore, the spatial frequency content of these superstructures can be approximated by a comb of delta functions centered at the Bragg frequency. The separation between consecutive peaks can be controlled by changing the sampling period, $M$, and is given by [37]

$$
\Delta \lambda=\frac{\lambda_{B}^{2}}{2 n_{\mathrm{eff}} M} .
$$

Taking this into account, several experiments were performed: first by amplitude modulation, and then by phase modulation, allowing dense channel spacing without increasing the total grating length.

Amplitude modulation was performed by two ways: by changing the acousto-optic cell diffraction efficiency and by changing the phase mask dithering amplitude. Figure 17 shows results of two sampled Bragg gratings written by changing the cell efficiency from 0 to $85 \%$ (maximum attainable) with two different sampling periods. Both 30-mm-length Bragg gratings shown were written in the hydrogen loaded standard single mode fiber (ITU-T G.652) using a scanning beam velocity of $30 \mu \mathrm{m} / \mathrm{s}$.

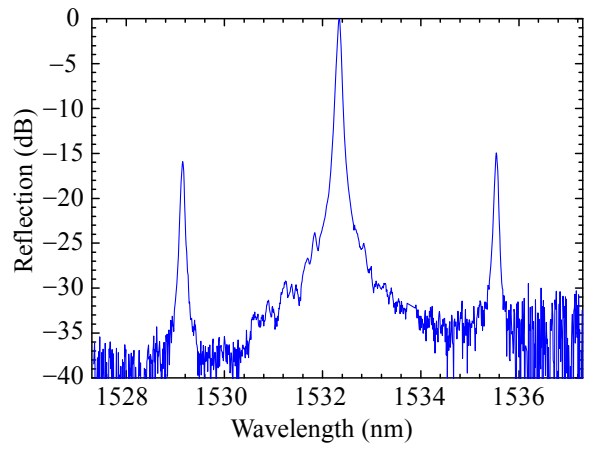

(a)

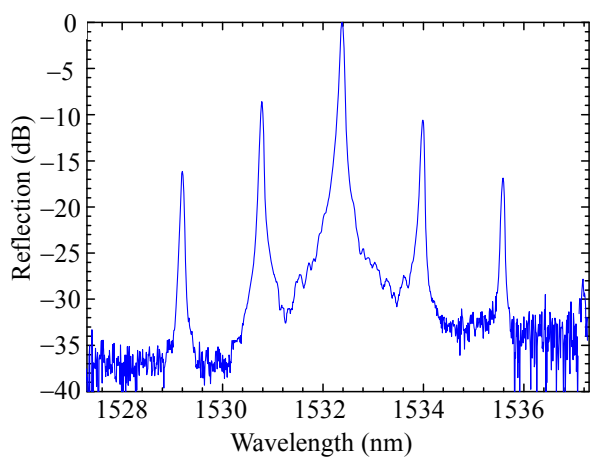

(b)

Fig. 17 Sampled fiber Bragg gratings fabricated by amplitude modulation through acousto optic cell efficiency variation $(0,85 \%, 0,85 \%, \cdots)$ with a sampling period of (a) $250 \mu \mathrm{m}$ and (b) $500 \mu \mathrm{m}$.

A sampling period of $250 \mu \mathrm{m}$ and $500 \mu \mathrm{m}$ should result in a peak separation of $3.24 \mathrm{~nm}$ and $1.62 \mathrm{~nm}$, respectively, as predicted by using (9). From Fig. 17(a), a peak separation of $3.19 \mathrm{~nm}$ is measured, while from Fig. 17(b) a value of $1.60 \mathrm{~nm}$ is obtained. This shows good agreement with predicted values and confirms that by doubling the sampling period one can easily halve the peak spacing. Another way of modulating the refractive index amplitude is by phase mask dithering, by changing visibility from 0 to 1 with a well defined sampling period. Figure 18 shows an example of a sampled Bragg grating written by this method. By using this technique, only the visibility is changed, maintaining the average refractive index constant. The 30-mm-length fiber Bragg grating was also written in the hydrogen loaded standard single mode fiber (ITU-T G.652) with a scanning beam velocity of $30 \mu \mathrm{m} / \mathrm{s}$ and a period of $500 \mu \mathrm{m}$, resulting in a 
measured peak separation of $1.60 \mathrm{~nm}$.

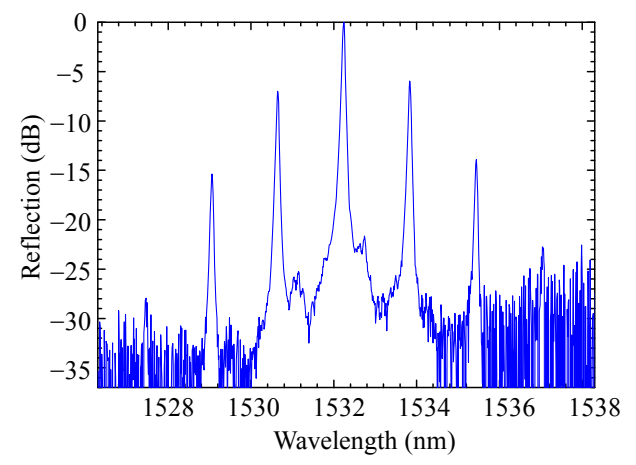

Fig. 18 Sampled fiber Bragg grating fabricated by amplitude modulation through phase mask dithering variation (visibility: 0 , $1,0,1, \cdots)$ with a sampling period of $500 \mu \mathrm{m}$.

However, the most interesting approach is grating sampling by using multiple phase shifts, which can realize dense channel spacing without increasing the total grating length [38]. Using this approach, peak equalization was also sought. Figure 19 illustrates the results obtained with several discrete $\pi$ phase shifts. The final sampled fiber Bragg grating spectrum shown in Fig. 19 (solid line) was achieved by a double exposure process. In the first exposure (dashed line), a UV beam power of about $80 \mathrm{~mW}$ was scanned at $20 \mu \mathrm{m} / \mathrm{s}$, and a sampling period of $500 \mu \mathrm{m}$ was employed, resulting in a peak separation of $1.6 \mathrm{~nm}$ [confirmed by (9)]. In the second exposure, a UV beam power of about $60 \mathrm{~mW}$ was scanned at $30 \mu \mathrm{m} / \mathrm{s}$, and a sampling period of $1000 \mu \mathrm{m}$ was employed, resulting in a peak separation of $0.8 \mathrm{~nm}$ [confirmed by (9)].

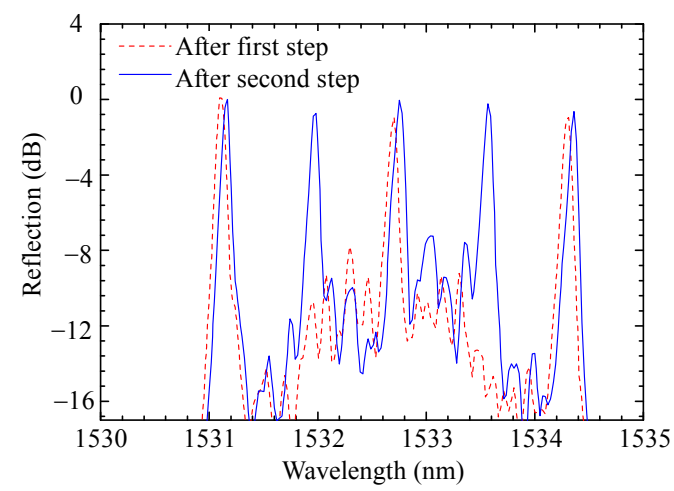

Fig. 19 Experimental results from sampled fiber Bragg gratings fabricated by phase modulation $(0, \pi, 0, \pi, \cdots)$ with a double exposure and different sampling periods.
In this way, new peaks appeared in the middle of the ones created by the first exposure grating since the sampling period doubled. By a suitable adjustment of exposure powers and scanning velocity, equalization was achieved, being the five peaks within $1.5-\mathrm{dB}$ peak power variation. The total grating length was $30 \mathrm{~mm}$ and was fabricated in the hydrogen loaded standard single mode fiber (ITU-T G.652).

The system developed also included a holder for planar integrated optic devices, and therefore all results demonstrated here on fibers were also obtained in planar devices. The main difference was related to the fact that a charge coupled device (CCD) camera was used to align the waveguides to the UV writing beam.

\section{Conclusions}

Most of the methods currently available for grating fabrication are based on the phase mask technique and allow the fabrication of good quality and reproducible Bragg gratings, however with very low flexibility. This means that if other types of gratings are required, the use of a phase mask with the desired profile is also necessary, increasing the cost/effectiveness of the method. Thus, the method chosen in this work made use of the effectiveness of the phase mask method, introducing other control elements to allow the tailoring of Bragg grating structures. The phase mask dithering/moving technique allows, in this way, the combination of simplicity and high reproducibility with the desired flexibility. Some of the essential aspects related to the experimental implementation of this method were presented in this work. Basically, the local grating visibility or contrast and phase were controlled by the dithering/displacement of the phase mask, without affecting the average refractive index. High dithering amplitudes destroyed the interference pattern created by the phase mask at the fiber plane by fringe blurring. Thus, dithering is an ideal mechanism for varying the local strength of the 
grating without changing its local Bragg wavelength, since it keeps the average refractive index constant. Associated with the fact that tailored Bragg gratings can be achieved by using a standard uniform phase mask makes this one of the most interesting and useful methods available.

Different apodization profiles were compared, several phase shifted gratings were written, chirped behavior was achieved, and finally, sampled Bragg gratings by amplitude and phase modulation were shown. Another particularity of the developed setup is that it can be easily adapted to write Bragg grating in planar structures.

\section{Acknowledgment}

The authors thank Dr. Isabelle Riant from Alcatel and Prof. Marc Douay from Lille University for their support that made this work possible. Also financial support from the European project PLATON - Planar Technology for Optical Networks (IST-2002-38168) is acknowledged. Prof. Ernst Brinkmeyer, Dr. Hagen Renner, and Frank Knappe are thanked for supplying the grating modulation profiles and performing the OFDR measurement. Multiwave Photonics, SA is acknowledged for making possible the dispersion measurement of the chirped gratings.

Open Access This article is distributed under the terms of the Creative Commons Attribution License which permits any use, distribution, and reproduction in any medium, provided the original author(s) and source are credited.

\section{References}

[1] K. O. Hill, Y. Fujii, D. C. Johnson, and B. S. Kawasaki, "Photosensitivity in optical fiber waveguides: application to reflection filter fabrication," Applied Physics Letters, vol. 32, no. 10, pp. 647-649, 1978.

[2] G. Meltz, W. W. Morey, and W. H. Glenn, "Formation of Bragg gratings in optical fibers by a transverse holographic method," Optics Letters, vol. 14, no. 15, pp. 823-825, 1989.

[3] H. M. Philips, D. L. Callahan, R. Sauerbrey, G. Szabó, and Z. Bor, "Sub-100 nm lines produced by direct laser ablation in polyimide," Applied Physics Letters, vol. 58, no. 24, pp. 2761-2763, 1991.

[4] P. E. Dyer, R. J. Farley, and R. Giedl, "Analysis and application of a $0 / 1$ order Talbot interferometer for $193 \mathrm{~nm}$ laser grating formation," Optics Communications, vol. 129, no. 1-2, pp. 98-108, 1996.

[5] K. O. Hill, B. Malo, K. A. Vineberg, F. Bilodeau, D. C. Johnson, and I. Skinner, "Efficient mode conversion in telecommunication fiber using externally written gratings," Electronics Letters, vol. 26, no. 16, pp. 1270-1272, 1990.

[6] B. Malo, K. O. Hill, F. Bilodeau, D. C. Johnson, and J. Albert, "Point-by-point fabrication of micro-Bragg gratings in photosensitive fiber using single excimer pulse refractive index modification techniques," Electronics Letters, vol. 29, no. 18, pp. 1668-1669, 1993.

[7] A. Martinez, M. Dubov, I. Khrushchev, and I. Bennion, "Direct writing of fiber Bragg gratings by femtosecond laser," Electronics Letters, vol. 40, no. 19, pp. 1170-1172, 2004.

[8] G. D. Marshall, R. J. Williams, N. Jovanovic, M. J. Steel and M. J. Withford, "Point-by-point written fiber-Bragg gratings and their application in complex grating designs," Optics Express, vol. 18, no. 19, pp. 19844-19859, 2010.

[9] R. J. Williams, C. Voigtlander, G. D. Marshall, A. Tünnermann, S. Nolte, M. J. Steel, et al., "Point-by-point inscription of apodized fiber Bragg gratings," Optics Letters, vol. 36, no. 15, pp. 2988-2990, 2011.

[10] K. O. Hill, B. Malo, F. Bilodeau, D. C. Johnson, and J. Albert, "Bragg gratings fabricated in monomode photosensitive optical fiber by UV exposure through a phase mask," Applied Physics Letters, vol. 62, no. 10, pp. 1035-1037, 1993.

[11] D. Z. Anderson, V. Mizrahi, T. Erdogan, and A. E. White, "Production of in-fiber gratings using a diffractive optical-element," Electronics Letters, vol. 29, no. 6, pp. 566-568, 1993.

[12] J. Martin and F. Ouellette, "Novel writing technique of long and highly reflective in-fiber gratings," Electronics Letters, vol. 30, no. 10, pp. 811-812, 1994.

[13] B. Malo, D. C. Johnson, F. Bilodeau, J. Albert, and K. O. Hill, "Single-excimer-pulse writing of fiber gratings by use of a zero-order nulled phase mask: grating spectral response and visualization of index perturbations," Optics Letters, vol. 18, no. 15, pp. 1277-1279, 1993.

[14] Qin Zhang, D. A. Brown, L. Reinhart, T. F. Morse, J. Q. Wang, and G. Xiao, "Tuning Bragg wavelength by writing gratings on prestrained fibers," IEEE 
Photonics Technology Letters, vol. 6, no. 7, pp. 839-841, 1994.

[15] M. Matsuhara and K. O. Hill, "Optical waveguide band-rejection filters - design," Applied Optics, vol. 13, no. 12, pp. 2886-2888, 1974.

[16] K. Tanaka, K. Sakamoto, T. Tanaka, and M. Taguchi, "Apodization for Gaussian-beam incidence numerical examination," Optics Communications, vol. 115, no. 1-2, pp. 29-34, 1995.

[17] B. Malo, S. Theriault, D. C. Johnson, F. Bilodeau, J. Albert, and K. O. Hill, "Apodised in-fiber Bragg gGrating reflectors photoimprinted using a phase mask," Electronics Letters, vol. 31, no. 3, pp. 223-225, 1995.

[18] J. Albert, K. O. Hill, B. Malo, S. Theriault, F. Bilodeau, D. C. Johnson, et al., "Apodization of the spectral response of fiber Bragg gratings using a phase mask with variable diffraction efficiency," Electronics Letters, vol. 31, no. 3, pp. 222-223, 1995.

[19] P. Y. Cortes, F. Ouellette, and S. LaRochelle, "Intrinsic apodisation of Bragg gratings written using UV-pulse interferometry," Electronics Letters, vol. 34, no. 4, pp. 396-397, 1998.

[20] J. Albert, K. O. Hill, D. C. Johnson, F. Bilodeau, and M. J. Rooks, "Moire phase masks for automatic pure apodisation of fiber Bragg gratings," Electronics Letters, vol. 32, no. 24, pp. 2260-2261, 1996.

[21] R. Kashyap, A. Swanton, and D. J. Armes, "Simple technique for apodising chirped and unchirped fiber Bragg gratings," Electronics Letters, vol. 32, no. 13, pp. 1226-1228, 1996.

[22] M. J. Cole, W. H. Loh, R. I. Laming, M. N. Zervas, and S. Barcelos, "Moving fiber/phase mask-scanning beam technique for enhanced flexibility in producing fiber gratings with uniform phase mask," Electronics Letters, vol. 31, no. 17, pp. 1488-1490, 1995.

[23] W. H. Loh, M. J. Cole, M. N. Zervas, S. Barcelos, and R. I. Laming, "Complex grating structures with uniform phase masks based on the moving fiber-scanning beam technique," Optics Letters, vol. 20, no. 20, pp. 2051-2053, 1995.

[24] H. Hosono, Y. Abe, D. L. Kinser, R. A. Weeks, K. Muta, and H. Kawazoe, "Nature and origin of the 5-eV Band in $\mathrm{SiO}_{2}: \mathrm{GeO}_{2}$ Glasses," Physics Review B, vol. 46, no. 18, pp. 11445-11451, 1995.

[25] D. P. Hand and P. S. J. Russel, "Photoinduced refractive-index changes in germanosilicate fibers," Optics Letters, vol. 15, no. 2, pp. 102-104, 1990.

[26] M. Kristensen, "Ultraviolet-light-induced processes in germanium-doped silica," Physical Review B (Condensed Matter and Materials Physics), vol. 64, no. 14, pp. 4201-4212, 2001.

[27] T. Erdogan, "Fiber grating spectra," Journal of Lightwave Technology, vol. 15, no. 8, pp. 1277-1294, 1997.

[28] H. N. Rourke, S. R. Baker, K. C. Byron, R. S. Baulcomb, S. M. Ojha, and S. Clements, "Fabrication and characterisation of long, narrowband fiber gratings by phase mask scanning," Electronics Letters, vol. 30, no. 16, pp. 1341-1342, 1994.

[29] A. W. Snyder and J. D. Love, Optical Waveguide Theory, Dordrecht, The Netherlands: Kluwer Academic, 1983.

[30] J. Skaar and K. M. Risvik, "A genetic algorithm for the inverse problem in synthesis of fiber gratings," Journal of Lightwave Technology, vol. 16, no. 10, pp. 1928-1932, 1998.

[31] G. Cormier, R. Boudreau, and S. Thériault, "Real-coded genetic algorithm for Bragg grating parameter synthesis," Journal of the Optical Society of America B, vol. 18, no. 12, pp. 1771-1776, 2001.

[32] R. Feced, M. N. Zervas, and M. A. Muriel, "An efficient inverse scattering algorithm for the design of nonuniform fiber Bragg gratings," IEEE Journal of Quantum Electronics, vol. 35, no. 8, pp. 1105-1115, 1999.

[33] L. Poladian, "Simple grating synthesis algorithm," Optics Letters, vol. 25, no. 11, pp. 787-789, 2000.

[34] J. Skaar, L. Wang, and T. Erdogan, "On the synthesis of fiber Bragg gratings by layer peeling," IEEE Journal of Quantum Electronics, vol. 37, no. 2, pp. 165-173, 2001.

[35] S. Kieckbusch, C. Knothe, and E. Brinkmeyer, "Fast and accurate characterization of fiber Bragg gratings with high spatial and spectral resolution," presented at Conference on Optical Fiber Communication, Atlanta, USA (paper WL2), March 23, 2003.

[36] D. Derickson, Fiber Optic Test and Measurement. Englewood Cliffs, NJ: Prentice Hall, 1998.

[37] B. J. Eggleton, P. A. Krug, L. Poladian, and F. Ouellette, "Long periodic superstructure Bragg gratings in optical fibers," Electronics Letters, vol. 30, no. 19, pp. 1620-1622, 1994.

[38] Y. Nasu and S. Yamashita, "Multiple phase-shift superstructure fiber Bragg grating for DWDM systems," Electronics Letters, vol. 37, no. 24, pp. 1471-1472, 2001. 